\section{La perception de la recherche chez les infirmières oeuvrant en oncologie clinique}

\author{
par Debra A. Bakker, Terry Trottier, Claire McChesney
}

\section{Abrégé}

La recherche fait partie intégrante du domaine des soins infirmiers en oncologie et est une des raisons pour lesquelles la pratique infirmière en oncologie est devenue une spécialité. Et pourtant, on ne sait pas grand chose sur les perceptions en matière de recherche des infirmières oeuvrant en oncologie en dépit de la publication toujours croissante de recherches infirmières. La présente étude se proposait donc d'examiner les perceptions en matière de recherche des infirmières oeuvrant en oncologie clinique et de cerner les facteurs qui influencent ces perceptions. Deux cent quatre-vingt trois infirmières autorisées dispensant des soins en oncologie aux patients de quelque 40 établissements de soins de santé du nord de I'Ontario ont participé à cette enquête. Les données ont été recueillies à l'aide d'un questionnaire élaboré par Alcock et al. (1990) qui se penchait sur les perceptions des infirmières vis-à-vis de la recherche, de leur rôle, de leur intérêt et de leur expérience en la matière ainsi que sur les attitudes relatives à la recherche dans leur établissement. Les résultats montrent que les répondantes accordent une grande valeur à la recherche infirmière et qu'elles pensent que les infirmières soignantes ont un rôle à jouer en recherche. Toutefois, les répondantes estiment que les infirmières ne jouissent pas, au niveau de leurs collègues ou de l'administration, d'un soutien suffisant en ce qui concerne leur participation aux activités de recherche. Par ailleurs, les résultats de cette étude indiquent que la formation influence les perceptions en matière de recherche des infirmières oeuvrant en oncologie clinique.

\section{Introduction}

On retrouve dans les écrits de nombreux articles soulignant I'importance de la recherche infirmière et des rapports qu'elle entretient avec la pratique clinique (Bochm, 1985; Christoph, 1988; Jacox, 1974; MacPhail, 1991; McClure, 1981; Mercer, 1984; Schotfeldt, 1992). Ces articles et autres dissertations décrivent la signification de la recherche pour les soins infirmiers et pour les infirmières. Ainsi, la recherche témoigne de la crédibilité de notre profession, de ses responsabilités et de ses contributions uniques aux soins aux patients et aux résultats de santé. Quant aux infirmières individuelles, la recherche leur procure un bagage de connaissances sur lesquelles elles se basent pour prendre des décisions cliniques réfléchies et rehausser les normes de pratique infirmière et de soins aux patients.

Le tour d'horizon des trois dernières décennies nous révèle que bien des progrès ont été réalisés dans le domaine de la recherche infirmière.
Les infirmières ont obtenu un accès élargi à des cours et à une formation axés sur la recherche grâce à l'établissement de programmes menant au baccalauréat et post-diplôme collégial ainsi que des programmes d'études de $2^{e}$ et $3^{e}$ cycles en soins infirmiers (MacPhail, 1991). La productivité de la recherche se manifeste par la publication d'un nombre croissant de recherches infirmières et la prolifération des conférences et congrès nationaux et internationaux portant sur la recherche infirmière. De plus, les associations professionnelles d'infirmières ont publié des énoncés soulignant les attentes qu'elles avaient pour l'ensemble de leurs membres en matière de recherche (American Nurses' Association [ANA], 1981; Association des infirmières et infirmiers du Canada [AIIC], 1984, 1985, 1990). Ces perspectives et ces nouvelles réalisations ont permis d'atteindre le but final, à savoir une pratique infirmière fondée sur les résultats de la recherche.

Cependant, on ignore si ces réalisations se sont accompagnées d'un développement parallèle de l'esprit de recherche chez les infirmières oeuvrant dans les milieux cliniques. Par "esprit de recherche", on entend "une approche critique et un questionnement de sa pratique personnelle, le désir et la capacité de découvrir les résultats de recherche les plus récents... et la capacité d'en évaluer la valeur pour des situations données et d'en faire l'application pertinente" (Royal College of Nursing, tel que cité par O'Brien \& Heyman, 1989, p. 393). Cet esprit de recherche est essentiel à la pleine réalisation, dans les milieux de travail infirmier, de la pratique fondée sur les résultats de la recherche. Pour ce faire, les infirmières doivent avoir conscience des avantages de la recherche pour la pratique et les soins aux patients et les apprécier à leur juste valeur. C'est pourquoi les attitudes des infirmières envers la recherche et la manière dont elles perçoivent leur rôle dans ce domaine sous-tendent le concept d'esprit de recherche.

\section{Recension des écrits}

Les écrits portant sur la profession et la recherche indiquent qu'un des facteurs importants de l'élaboration de programmes de recherche en soins infirmiers cliniques est l'engagement des infirmières oeuvrant en clinique à soutenir la recherche à titre de domaine valable des soins infirmiers. Des rapports non scientifiques publiés dans les années 1970 et au début des années 1980 révèlent qu'à cette époque, la recherche infirmière ne faisait pas partie des priorités des infirmières oeuvrant en clinique (Jacox, 1974; Ketefian, 1975; McClure, 1981; Rettig, 1980). Celles-ci voyaient un conflit de rôle entre dispenser les soins aux patients et faire de la recherche avec ces mêmes patients. En fait, une croyance répandue voulait que "I'infirmière soignante était la bonne infirmière" (Rettig, 1980, p. 1253). Peu d'infirmières issues du milieu de la pratique

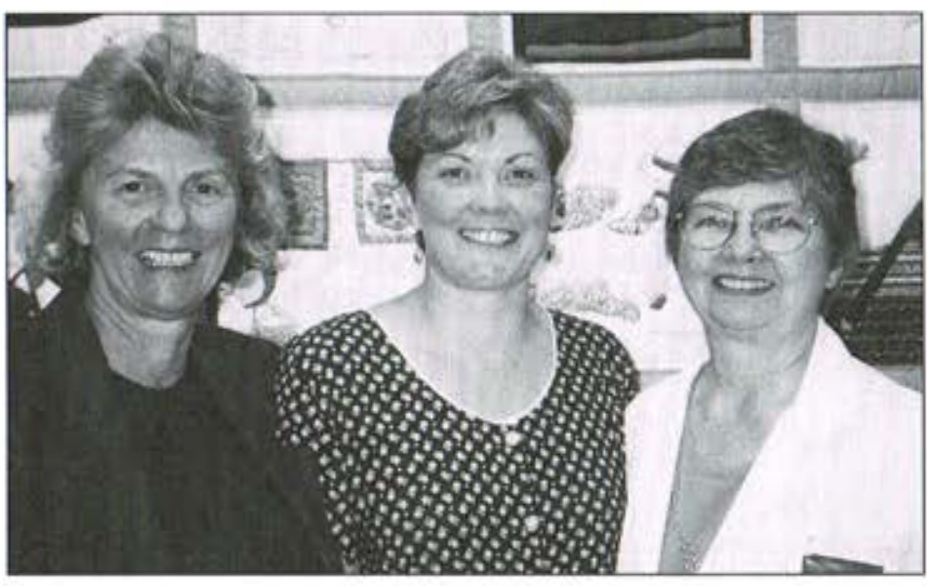

Degauche à droite: Claire McChesney, Debra Bakker et Terry Trottier

Debra A. Bakker, Inf, PhD est infirmière chercheure, Terry Trottier, Inf, est infirmière de soins intégraux en radiothérapie et Claire McChesney, Inf, $\mathrm{BScN}$, est responsable des programmes, oncologie préventive, au Centre régional de cancérologie du Nord-Est de l'Ontario, Sudbury, Ontario. 
reconnaissaient le lien entre la recherche et leur professionnalisation ou qu'elles pourraient jouer un rôle quelconque en vue de démontrer, grâce à la recherche, le fondement scientifique de la pratique infirmière (Boehm, 1985; McClure, 1981). Depuis cette époque, on mentionne les attitudes des infirmières soignantes comme étant l'un des principaux obstacles à la mise en oeuvre d'activités de recherche infirmière dans les milieux cliniques (Bowie, 1981; Davis, 1981; Egan, McElmurry \& Jameson, 1981; Noble, 1980; Oberst, 1985, Rettig, 1980).

Au cours des dix dernières années, des chercheurs de plusieurs pays ont réalisé des études visant à évaluer les attitudes des infirmières vis-à-vis de la recherche infirmière (Alcock, Carroll \& Goodman, 1990; Bostrom, Malnight, MacDougall \& Hargis, 1989; Chenitz, Satar \& Giefer, 1985; Ehrenfeld \& Eckerling, 1991; Marsh \& Brown, 1992; Poster, Betz \& Randell, 1992; Rizzuto, Bostrom, Suter \& Chenitz, 1994; Robichaud-Ekstrand \& Sherrard, 1994; Thomas, 1985; Webb \& MacKenzie, 1993). Dans l'ensemble, ces études montraient que les infirmières de la fin des années 80 et des années 90 entretenaient, à l'égard de la recherche, des attitudes plus positives que celles de leurs condisciples de la décennie précédente. De plus, on a découvert que l'engagement des infirmières oeuvrant en clinique en matière d'activités de recherche était surtout influencé par des facteurs personnels dont les attitudes, les croyances, l'expérience et la formation (Chenitz, Satar \& Giefer, 1985; Rizzutto et al., 1994).

Au Canada, quelques études ont êté effectuées en vue d'évaluer les perceptions des infirmières relatives à la recherche (Alcock et al., 1990; Robichaud-Ekstrand \& Sherrard, 1994; Varcoe \& Hilton, 1995). En 1988, Alcock et ses collègues ont mis au point un questionnaire permettant d'évaluer les attitudes des infirmières vis-à-vis de la recherche ainsi que le climat de recherche dans leur établissement. Les attitudes des infirmières y étaient conceptualisées sous la forme de croyances ou de sentiments sur la valeur de la recherche et sur celle du rôle des infirmières soignantes en matière de recherche et sous la forme d'énoncés comportementaux reflétant leur expérience et leur intérêt en matière de recherche. Quant au climat de recherche, on le retrouvait dans les perceptions portant sur l'appui de l'institution et la sensibilisation aux ressources offertes pour favoriser la recherche au sein de l'organisme de santé. Alcock et ses collègues (1990) ont utilisé la liste des membres de l'Ordre des infirmières et infirmiers de l'Ontario pour réaliser une enquête auprès d'un échantillon aléatoire d'infirmières autorisées oeuvrant dans divers milieux de pratique hospitalière et communautaire. Ils ont découvert que, bien que la majorité des infirmières exprimaient de l'intérêt pour la recherche, moins de $50 \%$ d'entre elles percevaient un soutien ou un encouragement en provenance de l'administration infirmière. De surcroît, certaines de ces infirmières (dans une proportion comprise entre 30 et $45 \%$ ) n'avaient pas été informées des ressources de recherche disponibles dans leur propre établissement.

Robichaud-Ekstrand \& Sherrard (1994) se sont servis du même questionnaire pour effectuer une enquête auprès des infirmières en cardiologie travaillant dans un hôpital d'enseignement du Québec. Les résultats obtenus montrent que ce groupe d'infirmières accordaient une grande valeur à la recherche infirmière surtout au niveau de ses rapports avec la pratique clinique.

La recherche a joué un rôle critique au niveau du développement de la masse des connaissances distinctes associćes aux soins infirmiers en oncologie, tout comme elle l'a fait pour les autres pratiques spécialisées. Toutefois, la recherche infirmière ne deviendra une réalité des soins infirmiers en oncologie que si toutes les infirmières en oncologie intériorisent la valeur de la recherche et favorisent l'avènement de la pratique fondée sur des résultats de recherche. De plus, à la lumière des changements actuels vécus dans le système des soins de santé en vertu desquels tous les professionnels de la santé doivent examiner leur pratique et justifier leur rôle au sein du système, le développement de la composante recherche se fait encore plus pressant. C'est dans le contexte de ces efforts que vient s'inscrire la présente enquête portant sur les attitudes relatives à la recherche des infirmières en oncologie oeuvrant dans divers milieux cliniques.

Jusqu'à présent, on ne sait que peu de choses sur les perceptions des infirmières sur la recherche ou sur les rôles qu'elles prétendent jouer dans ce domaine. On n'a pas repéré, dans les écrits, une seule étude de mesure des perceptions des infirmières en oncologie relatives à la recherche. La présente étude avait donc pour objet d'examiner les perceptions en matière de recherche des infirmières oeuvrant en oncologie clinique et de déterminer les facteurs qui influent sur leurs perceptions.

\section{Méthodologie \\ Échantillon et milieu}

On a recensé comme sites potentiels de recherche tous les établissements de soins de santé du nord de l'Ontario entretenant des liens plus ou moins étroits avec les centres régionaux de cancérologie. On s'est assuré la collaboration des coordinatrices des services d'extension des deux centres régionaux de cancérologie du nord ontarien pour identifier les services infirmiers dispensant des soins aux patients atteints de cancer dans 43 établissements de soins. On estime à 444 le nombre d'infirmières autorisées oeuvrant dans ces services. On a invité l'ensemble de ces infirmières autorisées à participer à l'étude.

\section{Collecte des données}

On a recueilli les données relatives aux perceptions des infirmières en matière de recherche au moyen du questionnaire d'auto-évaluation conçu par Alcock et al. (1990). Le questionnaire se compose de cinq sections qui portent sur la problématique suivante: 1) la perception de la valeur de la recherche infirmière; 2) la perception du rôle dans la recherche; 3) l'intérêt pour la recherche; 4) le climat de recherche dans l'organisme qui comprend aussi le soutien perçu pour la recherche infirmière et la prise de conscience de l'existence des ressources offertes à l'appui de cette recherche. On a également recueilli des données de nature démographique telles que l'âge, la situation d'emploi, le type de formation infirmière, le nombre d'années depuis la fin des études ainsi que les caractéristiques du milieu de travail.

Dans le cas des sections concernant la valeur, le rôle, l'intérêt et le climat de recherche de l'établissement, les répondantes estimaient les items en fonction d'une échelle de Likert à 4 points où les réponses allaient de "tout à fait d'accord" à "pas du tout d'accord", Quant aux items portant sur l'expérience de recherche des infirmières, on y répondait par "oui" ou "non" tandis que pour les énoncés ayant trait à la sensibilisation aux ressources offertes à l'appui de la recherche, on devait choisir parmi les réponses "oui", "non", ou "je ne sais pas".

La validité du contenu du questionnaire avait déjà été mise à l'épreuve par des infirmières expertes des milieux cliniques et par des infirmières chercheures. L'analyse de fidélité des scores composés obtenus pour chaque section révélait un fort indice de cohérence interne parmi les items de chacune des cinq sections avec des coefficients supérieurs à 0,71 (Alcock et al., 1990). D'autres tests psychométriques appliqués au questionnaire dans le cadre de l'étude de Robichaud-Ekstrand \& Sherrard (1994) ont démontré la validité des construits et la validité concomitante.

Les personnes-contacts de 43 établissements ont distribué les trousses du questionnaire à toutes les infirmières autorisées travaillant dans les services de soins identifiés. On demandait aux infirmières de remplir le questionnaire sans le signer et de le renvoyer directement aux chercheures en utilisant l'enveloppe adressée et affranchie jointe.

\section{Analyse des données}

On a procédé à l'analyse des données en calculant la fréquence du taux de réponse pour chacun des énoncés et en estimant un score moyen pour chacune des cinq variables, à savoir la valeur, le rôle, l'expérience, l'intérêt et le climat de recherche. En vue de cerner les facteurs qui influent sur les perceptions des infirmières en matière de recherche, on a effectué des analyses de variance univariée ou des 
tests de $t$ (au moyen d'un alpha de 0,05 ) pour les cinq scores moyens en fonction de l'âge, de la situation d'emploi, des années depuis la fin des études, de la formation infirmière, du lieu de travail et de l'emplacement de l'institution.

\section{Résultats}

Caractéristiques de l'échantillon

En tout, on a distribué 444 questionnaires à 43 organismes. Deux cent quatre-vingt-trois infirmières représentant 40 organismes ont retourné le questionnaire dûment rempli pour un taux de réponse de l'ordre de $64 \%$. On n'a rien reçu de trois établissements auxquels on avait fait parvenir un total de 19 questionnaires. Le tableau I décrit les caractéristiques des répondantes. Cinquante pour cent des infirmières avaient 40 ans ou plus, $70 \%$ travaillaient à temps plein, $71 \%$ avaient terminé leurs études depuis plus de dix ans et $79 \%$ avaient reçu leur formation infirmière dans des collèges communautaires ou dans le cadre de programmes hospitaliers de sciences infirmières. Pour ce qui est du milieu de travail, $46 \%$ des répondantes dispensaient des soins à des hospitalisés. Quant à la distribution entre établissements urbains et ruraux, l'échantillon était raisonnablement équilibré puisqu'on comptait $54 \%$ d'établissements urbains contre $46 \%$ de ruraux. Pour ce qui est de notre étude, on a désigné comme étant urbaines toutes les institutions situées dans des villes dont la population était égale ou supérieure à 40000 habitants.

Afin de savoir dans quelle mesure les répondantes du nord ontarien reflétaient la population globale des infirmières du Canada, on a comparé les données démographiques (âge, formation et situation d'emploi) aux données de gestion sur les infirmières autorisées compilées par Statistique Canada (1993) (voir le tableau 2). Quand on a comparé l'échantillon de l'étude aux infirmières autorisées inscrites en Ontario et au Canada, on a remarqué qu'il y avait une plus forte proportion d'infirmières dans le plus jeune groupe d'âge et une plus petite dans le plus vieux groupe d'âge $(p<0,05)$. La comparaison des données de situation d'emploi a révélé que la distribution de $70 \%$ d'infirmières à temps plein et de $30 \%$ à temps partiel différait de manière significative de la proportion qui prévaut

Tableau 1: Caractéristiques de l'échantillon $(\mathrm{N}=283)$

$\begin{array}{lrr}\text { Variables démographiques } & \text { Nombre } & \% \\ \text { Groupe d'âge } & & \\ \leq 29 & 47 & 16 \\ 30-39 & 96 & 34 \\ 40-49 & 101 & 36 \\ 50 \text { ct plus } & 39 & 14\end{array}$

Situation d'emploi

Temps plein

Temps partiel

Fins des études

10 ans ou moins

Plus de 10 ans

201

29

Formation

Diplôme/Certificat

BSclnf

Milieu de travail

Services aux hospitalisés

Consultations externes

Combinaison des deux items ci-dessus

57

Soins communautaires

31

Milieu de l'institution

Milieu urbain

152

Milicu rural actuellement dans les populations d'infirmières de l'Ontario et du Canada $(\mathrm{p}<0,05)$. On remarquait également une différence statistiquement significative au niveau de la proportion des infirmières préparées au niveau du diplôme par rapport à celles ayant obtenu un baccalauréat en sciences infirmières lorsqu'on les comparait aux infirmières inscrites en Ontario ( $<<0,05)$. En effet, les répondantes étaient plus nombreuses à avoir obtenu leur BScInf. Par contre, lors de leur comparaison aux infirmières canadiennes, le test de concordance du niveau de formation n'a pas donné de résultats significatifs à $\mathrm{p}<0,05$.

\section{Perceptions de la recherche}

Le tableau 3 illustre le score moyen correspondant à chacune des cing variables calculées pour l'ensemble des infirmières en oncologie de l'échantillon. Les scores élevés traduisent une perception plus positive de la variable.

Tableau 2: Comparaison des infirmières en oncologie avec a) les infirmières autorisées de l'Ontario et b) les infirmières autorisées du Canada

\section{A. Groupe d'infirmières}

\begin{tabular}{ccc} 
& \multicolumn{2}{c}{$\hat{A} g e$ (années) } \\
529 & $30-39$ & $40-49$
\end{tabular}

Infirmières en oncologie $(\mathrm{N}=281)$

Infirmières autorisées de I'Ontario ( $\mathrm{N}=84,343)$

Infirmières autorisées du Canada $(\mathrm{N}=235,630)$

\section{B. Groupe d'infirmières}

$16 \% \quad 34 \% \quad 36 \%$

$13 \% * \quad 30 \% \quad 34 \%$ $14 \%$

$14 \% *$

$32 \%$

$33 \%$ $23 \% *$

$21 \% *$

Situation d'emploi

Tempsplein Tempspartiel/ Travail occasionnel

Infirmières en oncologie $(\mathrm{N}=281)$

Infirmières autorisées de l'Ontario ( $\mathrm{N}=84,343)$

Infirmières autorisées du Canada $(\mathrm{N}=235,630)$

C. Groupe d'infirmières $70 \%$ $30 \%$

$57 \% *$

$43 \% *$

$59 \% *$

$41 \%{ }^{*}$

Infirmières en oncologie $(\mathrm{N}=279)$

Infirmières autorisées de l'Ontario $(\mathrm{N}=84,343)$

Infirmières autorisées du Canada $(\mathrm{N}=235,630)$

${ }^{*} \mathrm{p}<0.05$

\section{Formation}

Diplôme BSeInf

$\begin{array}{ll}79 \% & 21 \% \\ 84 \% * & 16 \% * \\ 83 \% & 17 \%\end{array}$

Tableau 3: Perceptions des infirmières en oncologie vis-at-vis de la recherche $(\mathrm{N}=283)$

$\begin{array}{lcr}\text { Variable } & \text { Score moyen } & \begin{array}{r}\text { Intervalle } \\ \text { des scores }\end{array} \\ \text { Valeur } & 15.5(2.1) & 5-20 \\ \text { Róle } & 31.8(3.7) & 10-40 \\ \text { Intérêt } & 24.0(2.5) & 8-32 \\ \text { Expérience } & 2.8(2.5) & 0-13 \\ \text { Climat de recherche } & 23.0(3.7) & 9-36\end{array}$


Valeur de la recherche:

Le score moyen de la valeur indique que les infirmières de cette étude considèrent, dans leur ensemble, que la recherche est de grande valeur et qu'elle s'applique à la pratique infirmière (voir le tableau 3 ). Quatre-vingt-quinze pour cent des répondantes étaient d'accord pour dire que les connaissances fondées sur la recherche aident les infirmières à résoudre des problèmes de soins aux patients et que la recherche permet d'améliorer la pratique clinique. En outre, $88 \%$ d'entre elles indiquaient que les résultats de recherche leur fournissent les "données" dont elles ont besoin pour prendre des décisions cliniques tandis qu'elles reconnaissaient (dans de plus faibles pourcentages) que la recherche est rentable ( $79 \%$ ) et qu'elle favorise la responsabilisation vis-à-vis de la pratique ( $70 \%)$.

Le rôle des infirmières soignantes dans la recherche:

Le score moyen pour cet item révèle que les répondantes acceptent que la recherche fasse partie des responsabilités de l'infirmière soignante (voir le tableau 3 ). Cette perception se reflète le plus nettement dans les réponses des infirmières qui sont d'accord pour dire que les infirmières soignantes devraient jouer un rôle au niveau de l'identification des problèmes de soins infirmiers ( $99 \%$ ), trouver des moyens de résoudre les problèmes de soins infirmiers $(99 \%)$, suggérer des façons d'améliorer les soins aux patients (100\%) et appliquer les résultats de la recherche dans leur pratique infirmière (96\%). Et bien que la quasi-totalité des infirmières $(97 \%)$ indiquaient qu'elles se voyaient jouer un rôle précis en matière de recherche qui viserait à améliorer la qualité des soins infirmiers, elles étaient moins nombreuses à accepter le fait que les infirmières soignantes devraient s'occuper de recueillir des données pour des études de recherche infirmière ( $88 \%$ ) ou d'effectuer ces mêmes études (60\%). Et la moyenne baisse à $50 \%$ lorsqu'il s'agit de recueillir des données pour des études autres que les études en soins infirmiers.

Le score moyen correspondant à la variable intérêt indique que les répondantes s'intéressent à la recherche (voir le tableau 3 ). Toutefois, l'analyse des items individuels du questionnaire révèle que cet intérêt semble refléter la quantité de participation active requise de l'infirmière. Ainsi, les infirmières s'intéressaient beaucoup au fait de trouver une réponse à des problèmes de pratique particuliers $(95 \%)$ ou de connaître les résultats de projets de recherche réalisés dans leur service $(95 \%)$. La rédaction de ces items est telle qu'on n'y exige pas une action spécifique de la part des infirmières. Le pourcentage des répondantes intéressées diminuait lorsque les items indiquaient un engagement plus actif de l'infirmière. Par exemple, $82 \%$ des infirmières ont signalé l'intérêt qu'elles avaient de participer à des projets de recherche dans leur service; $74 \%$ ont dit que la lecture d'études de recherche les intéressait alors que $58 \%$ exprimait de l'intérêt pour faire partie d'un comité de recherche.

\section{Expérience de recherche}

Les infirmières du groupe concerné par l'étude possédait très peu d'expérience de recherche (voir le tableau 3). Environ un quart des

Tableau 4: Différences significatives dans les perceptions des infirmières en oncologie vis-à-vis de la recherche, en fonction de la formation

Variable

$$
\text { Diplôme ( }=220)
$$
Score moyen

$$
\begin{array}{lr}
\text { Valeur** } & 15.3(2.1) \\
\text { Rôle* } & 31.4(3.6) \\
\text { Intérêt* } & 23.6(4.7) \\
\begin{array}{l}
\text { Expérience** } \\
\text { Climat }
\end{array} & 2.0(1.9) \\
\quad \text { de recherche } & 22.9(3.7)
\end{array}
$$

Formation

BScInf $(\mathrm{N}=59)$ Score moyen répondantes ont signalé avoir suivi un cours de recherche, élaboré un problème ayant fait l'objet d'une recherche par la suite, dirigé des entrevues de recherche, recueilli des échantillons pour la recherche ou encore assisté à une conférence de recherche. Les activités de recherche qu'elles mentionnaient le plus souvent étaient de modifier la pratique infirmière en fonction des résultats de la recherche $(48 \%)$ et de remplir des questionnaires dans le cadre de projets de recherche (71\%).

\section{Climat de recherche}

Le score moyen ayant trait au climat de recherche indiquait que, selon les répondantes, le soutien était plutôt modeste dans leur institution (voir le tableau 3). En ce qui concerne le soutien de l'administration, plus de la moitié des répondantes (soit $62 \%$ ) ont signalé que l'administration infirmière appuyait les infirmières engagées dans des projets de recherche, mais moins de la moitié d'entre elles déclaraient que les infirmières qui participaient aux activités de recherche voyaient leurs efforts reconnus (49\%), ou bien qu'elles étaient encouragées par l'administration infirmière à questionner leur pratique $(43 \%)$ ou à mettre au point des méthodes de pratique plus efficaces et efficientes par le biais de recherche (43 $\%)$. En ce qui concerne le soutien collaboratif, les infirmières de l'échantillon ne percevaient pas beaucoup de soutien de la part de leurs collègues, car elles n'étaient que $28 \%$ à dire que les infirmières soutiennent leurs collègues participant à des projets de recherche. En réalité, les résultats indiquaient que les collègues de travail autres que les infirmières offraient un meilleur soutien. Par exemple, $45 \%$ des répondantes estimaient que les médecins soutenaient la recherche infirmière et $71 \%$ jugeaient que les professionnels des autres disciplines (diététistes et travailleuses sociales) étaient prêtes à collaborer à des projets de recherche. Au moins $20 \%$ des répondantes n'ont pas complété les items se rapportant au rôle des professeures d'université en sciences infirmières à titre de ressources ou de collaboratrices. Cette proportion importante de valeurs manquantes reflète probablement l'absence de contact ou les contacts limités entre les institutions du nord ontarien et les écoles en sciences infirmières universitaires.

En plus des perceptions des infirmières sur le soutien apporté à la recherche dans le milieu de travail, on a évalué le climat de recherche en dégageant la prise de conscience des infirmières relative aux ressources disponibles dans leur institution. Les résultats démontrent qu'au moins 20 à $30 \%$ des membres de l'échantillon ne savaient pas du tout si de telles ressources y existaient. Les ressources disponibles prenaient les formes suivantes: $53 \%$ des répondantes ont indiqué que, dans leur institution, des services de recherche en bibliothèque étaient ouverts aux infirmières; $41 \%$ d'entre elles déclaraient être au courant de l'existence d'un comité d'éthique pour la recherche dans leur institution et $34 \%$ signalaient qu'une représentante de la profession infirmière y siégeait; $29 \%$ des répondantes indiquaient que des services informatiques étaient mis à leur disposition mais, seulement $15 \%$ ont indiqué que les infirmières désirant déposer des demandes de subventions et des propositions de recherche pouvaient bénéficier de services de secrétariat. Elles n'étaient que $21 \%$ à signaler la disponibilité, dans leur milieu de travail, de chercheures ou de personnes expérimentées qui pourraient les aider dans le cadre de l'analyse des données.

\section{Facteurs influençant les perceptions}

des infirmières en matière de recherche

En vue de déterminer si les facteurs de nature démographique influençaient les perceptions des infirmières vis-à-vis de la recherche, on a analysé les cinq scores du tableau 3 en fonction de l'âge, de la situation d'emploi, des années depuis la fin des études, du niveau de formation, du milieu de travail et de l'emplacement de l'institution (voir le tableau 4). Cette analyse a révélé qu'un seul de ces facteurs influait sur les perceptions des infirmières à l'égard de la recherche, à savoir le niveau de formation. On a découvert une différence statistiquement significative dans le cas des infirmières ayant obtenu un BScInf: celles-ci obtenaient de meilleurs résultats en matière de 
valeur, de rôle, d'intérêt et d'expérience de la recherche, mais pas en matière de climat de recherche.

\section{Discussion}

La recherche infirmière a pour but de fournir une base scientifique à la pratique infirmière. La recherche est l'instrument qui permet d'élaborer des stratégies de soins infirmiers à la fois efficaces et efficientes et de démontrer que les interventions infirmières font toute la différence au niveau des soins aux patients. Si on la considère sous cet angle, on peut avancer que la recherche infirmière n'en revêt que plus d'importance dans le contexte du systėme actuel des soins de santé caractérisé par le changement, la compression des ressources et une responsabilisation croissante des professionnels (Baumgart, 1995; Butler, 1995; Smeltzer \& Hinshaw, 1993; Wong, 1996). Cependant, si on veut atteindre le but visé, à savoir une pratique infirmière fondée sur des résultats de recherche, et utiliser la recherche comme vecteur du changement, il incombe aux infirmières à tous les niveaux de développer leur esprit de recherche. Une évaluation systématique des attitudes des infirmières à l'égard de la recherche renseigne sur l'esprit de recherche qu'elles manifestent et permet de dégager les facteurs qui favorisent ou bien freinent les efforts de recherche.

Les résultats globaux de cette étude suggèrent que les infirmières oeuvrant dans le domaine des soins infirmiers en oncologie sont de plus en plus convaincues de la valeur de la recherche infirmière. Les résultats montrent que, majoritairement, les infirmières de l'échantillon entretenaient des perceptions positives à l'égard de la recherche infirmière dont elles reconnaissaient la valeur dans le cadre de la pratique clinique. Elles ont également exprimé leur intérêt dans les activités de recherche et conçu un rôle de recherche pour les infirmières soignantes. Le niveau d'intérêt relatif à la recherche et le rôle perçu pour les infirmières soignantes rapportés par les répondantes rejoignaient les responsabilités de recherche définies pour les infirmières oeuvrant en clinique par les associations professionnelles d'infirmières (ANA, 1981; AIIC, 1984; 1985; 1990). Les répondantes ont nettement accepté les responsabilités attribuées aux infirmières soignantes au niveau de l'identification de problèmes pertinents en soins infirmiers et de l'utilisation, dans la pratique, des résultats de la recherche infirmière. On retrouve des perceptions similaires tout aussi positives dans les études canadiennes antérieures qui se servaient du même instrument de mesure pour examiner les attitudes envers la recherche des infirmières oeuvrant en clinique (Alcock et al., 1990; Robichaud-Ekstrand \& Sherrard, 1994; Varcoe \& Hilton, 1995). En outre, les recherches portant sur les attitudes des infirmières effectuées aux États-Unis, en Israęl et au Royaume-Uni au cours de la dernière décennie étayent le concept de l'intégration naissante de la recherche chez les infirmières dispensant des soins directs aux patients (Bostrom et al., 1989; Ehrenfeld \& Eckerling, 1991; Marsh \& Brown, 1992; Poster et al., 1992; Rizzuto et al., 1994; Thomas, 1985; Webb \& MacKenzie, 1993).

Bien que les résultats de cette étude révèlent une tendance positive vers l'adoption de l'esprit de recherche par les infirmières oeuvrant en clinique, les résultats dégagent également les facteurs freinant les efforts visant à combler l'écart entre la recherche et la pratique. En premier lieu, les répondantes ne percevaient pas de soutien collaboratif ou administratif évident pour les infirmières participant à des activités de recherche. Cette perception du manque de reconnaissance, par les collègues et l'administration, de l'importance des efforts de recherche en milieu clinique et du manque de soutien ont été signalés dans d'autres études (Alcock ct al., 1990; Bostrom et al., 1989; Bowie, 1981). De plus, une grande partie des infirmières de l'échantillon n'étaient pas au courant des ressources disponibles pour la recherche infirmière dans leur institution. Ce faible degré de sensibilisation reflète peut-être un des autres résultats de l'étude à savoir que la majorité des répondantes avaient une expérience limitée de la recherche et qu'elles ignoraient donc l'existence d'une telle infrastructure organisationnelle ou que celle-ci n'était pas utile à leurs yeux. Cependant, ce faible degré de sensibilisation pourrait aussi refléter la négligence de l'administration des soins de santé qui n'a pas pris les mesures nécessaires pour créer et favoriser des milieux de pratique fondée sur les résultats de la recherche. Et bien que les infirmières oeuvrant en clinique se doivent d'assumer leur responsabilité professionnelle individuclle pour ce qui est de l'acquisition de connaissances fondées sur les résultats de la recherche, l'utilisation de la recherche ne pourra devenir une réalité que si les attitudes et les actions des infirmières individuelles trouvent un écho dans la culture organisationnelle qui doit favoriser et exiger une pratique infirmière fondée sur des résultats de recherche et ce, même en pleine réforme des soins de santé (Baumgart, 1996; Vail \& Hicks, 1996; Varcoe \& Hilton, 1995).

Un second point soulevé par les résultats de cette étude concerne les facteurs qui influent sur les perceptions des infirmières vis-à-vis de la recherche. L'étudé a démontré que la formation reçue influençait les perceptions relatives à la valeur, au rôle, à l'intérêt et à l'expérience en matière de recherche. Ainsi, les infirmières ayant obtenu un baccalauréat étaient plus positives dans leurs perceptions que ne l'étaient les infirmières diplômées des collèges. Il est possible que ce résultat reflète un biais d'échantillonnage puisque l'échantillon de l'étude renfermait une proportion plus élevée d'infirmières détentrices d'un baccalauréat que l'ensemble des infirmières autorisées de l'Ontario et du Canada. Le résultat pourrait aussi refléter l'exposition des infirmières à des cours de recherche et à des chercheures dans le cadre de leur programme de baccalauréat à l'université. Ce résultat peut ne pas surprendre, mais il convient de se demander comment on peut favoriser l'adoption d'un esprit de recherche par les membres de la profession infirmière. Si en tant que discipline formelle, les soins infirmiers accordent une grande valeur à la recherche, la perçoivent comme composante vitale de l'essor de la profession et en acceptent la masse de connaissances, comment les infirmières qui n'ont pas reçu de formation précise en matière de recherche feront-elles pour acquérir cet esprit de recherche? Doit-on supposer que cette attitude sera encouragée et développée dans le lieu de travail ou par le biais d'autres moyens? Des études antérieures démontraient, elles aussi, que le niveau de formation influençait les attitudes vis-à-vis de la recherche. Dans l'ensemble, les études démontrent que les infirmières ayant un meilleur niveau de formation sont plus positives dans leurs perceptions de la recherche infirmière (Alcock et al., 1990; Bostrom et al., 1989, Chenitz et al., 1985; Ehrenfeld \& Eckerling, 1991), mais des études récentes ont prouvé le contraire (Robichaud-Ekstrand \& Sherrard, 1994) ou que la formation avait une incidence limitée (Varcoe \& Hilton, 1995). Par exemple, les infirmières en cardiologie possédant un baccalauréat et ocuvrant dans un hôpital d'enseignement avaient une perception moindre de valeur de la recherche, estimaient que la recherche était une partie mineure de leur rôle et exprimaient moins d'intérêt pour la recherche que leurs collègues préparées au niveau du diplôme (Robichaud-Ekstrand \& Sherrard, 1994). Et bien que les auteurs ci-dessus n'échafaudent pas d'hypothèses sur ce résultat contraire aux attentes, il est possible que l'environnement d'un hôpital d'enseignement et/ou l'expertise associée à une spécialité clinique jouent un rôle au niveau de l'internalisation de la recherche par les infirmières. Quant à Varcoe et Hilton (1995) dont les résultats indiquent que la formation a une incidence limitée sur l'utilisation des résultats de la recherche par les infirmières oeuvrant en clinique, ils suggèrent qu'il se peut que la qualité ou la quantité de formation en matière de recherche que reçoit une infirmière et/ou les caractéristiques du lieu de travail soient des facteurs qui influent sur I'utilisation quotidienne de la recherche par les infirmières travaillant dans des milieux pratiques.

On a souvent répété que les attitudes négatives des infirmières à l'encontre de la recherche représentaient un obstacle pour qui voulait combler l'écart entre la recherche et la pratique. Cela dit, cette étude rejoint les résultats d'autres travaux récents sur les perceptions de la recherche et elle véhicule un message à l'intention des cliniciennes, chercheures et administratrices. Les infirmières qui dispensent des soins dans des milieux cliniques ont, en matière de recherche, des perceptions différentes de celles signalées $15-20$ ans plus tôt. De nos jours, les infirmières oeuvrant en clinique perçoivent un rôle de recherche pour l'infirmière soignante et reconnaissent la contribution de la recherche à la pratique des soins infirmiers. 
Les infirmières canadiennes qui rapportent de telles perceptions ne sont pas seulement employées dans de grands hôpitaux d'enseignement mais elles oeuvrent aussi dans des lieux de travail communautaires et dans des institutions situées à une distance respectable des grands centres d'enseignement et de recherche en sciences de la santé.

Dans le contexte des compressions budgétaires et de la réforme des soins de santé actuelles, il est facile d'ignorer le besoin qu'ont les infirmières de consacrer une partie de leurs activités à la recherche. Néanmoins, les infirmières engagées dans la prestation directe des soins aux patients jouent un rôle vital au niveau de la mise en oeuvre d'une pratique fondée sur les résultats de la recherche. Elles sont aux premières loges en ce qui concerne l'identification des questions pertinentes de recherche portant sur la pratique clinique et de l'application des résultats de la recherche qui peuvent rehausser la qualité des soins aux patients. En acceptant de produire cet effort de recherche, elles permettent de combler le fossé séparant la

\section{Bibliographie}

Alcock, D., Carroll, G., Goodman, M. (1990). Staff nurses' perceptions of factors influencing their role in research. The Canadian Journal of Nursing Research, 22(4):7-18.

American Nurses' Association, Commission on Nursing Research. (1981). Guidelines for the investigative function of nurses. Kansas City, Mo.: American Nurses' Association.

Association des infirmières et infirmiers du Canada (1984). Les impératifs de la recherche infirmière au Canada. Le plan quinquennal vers l'an 2000. Ottawa. Association des infirmières et infirmiers du Canada.

Association des infirmières et infirmiers du Canada (1985). Prise de position : La recherche en soins infirmiers. Ottawa. Association des infirmières et infirmiers du Canada.

Association des infirmières et infirmiers du Canada (1990). Les impératifs de la recherche infirmière au Canada. Les cinq prochaines années : 1990-1995. Ottawa. Association des infirmières et infirmiers du Canada.

Baumgart, A.J. (1996). Promoting nursing practice through nursing research. International Nursing Review, 43(2):45-48.

Butler, L. (1996). Deciding on research in times of fiscal restraint: Can nursing risk the cost? Canadian Oncology Nursing Journal, 6(4): 178-180.

Bochm, S. (1985). Research as a basis for changing nursing practice. Topics in Clinical Nursing, 7(2):39-44.

Bostrom, A.C., Malnight, M., MacDougall, J., Hargis, D. (1989). Staff nurses' attitudes toward nursing research: a descriptive survey. Journal of Advanced Nursing. 14:915-922.

Bowie, R. (1981). Implementing research in the clinical setting. Journal of the Association of Operating Room Nurses. 33:1075-1077.

Chenitz, W.C., Satar, B., Giefer, K. (1985). Nurses' attitudes toward research and the clinical setting as a research environment. Proceedings of the National Symposium of Nursing: Advancing Clinical Practice. Stanford University Press, Palo Alto, 45-59.

Christoph, S.B. (1988). Delineating the responsibilities for nursing research. Critical Care Nurse, 8(3):27-29.

Davis, M. (1981). Promoting nursing research in the clinical setting. Journal of Nursing Administration. 11:22-27.

Egan, E.C., McElmurry, B.J., Jameson, H.M. (1981). Practice-based research: assessing your department's readiness. Journal of Nursing Administration. 11:26-32.

Ehrenfeld, M., Eckerling, S. (1991). Perceptions and attitudes of registered nurses to research: a comparison with a previous study. Journal of Advanced Nursing. 16:224-232.

Jacox, A. (1974). Nursing research and the clinician. Nursing Outlook, 22(6):382-385.

Ketefian, S. (1975). Application of selected nursing research findings into nursing practice: A pilot study. Nursing Research, 24:89-92. recherche et la pratique. Ce sont là les raisons pour lesquelles il importe qu'on mesure les attitudes des infirmières à l'égard de la recherche et que les infirmières chercheures, enseignantes et administratrices s'efforcent de canaliser ces attitudes afin de prouver que le travail des infirmières fait toute la différence dans les soins aux patients.

\section{Remerciements}

Les auteures tiennent à remercier et à reconnaître l'aide des personnes et organismes suivants: la section du Nord-Est de l'Ontario des infirmières en oncologie pour son appui financier. Mary Jane Kurm et Jackie Thoms pour leur assistance au niveau de la sélection des sites et de l'échantillon, Petra DeMeyere pour son aide relative à la préparation du manuscrit et les personnes-ressources qui ont facilité la distribution du questionnaire dans les établissements sélectionnés.

MacPhail, J. (1991). Research-mindedness in the profession. In: Canadian Nursing: Issues and Perspectives, (eds) Kerr, J.R. \& MacPhail, J. Toronto: Mosby-Year Book Inc. pp. 104-117.

Marsh, G.W., Brown, T.L. (1992). The measurement of nurses' attitudes towards nursing research and the research environment in clinical settings. Journal of Clinical Nursing. 1:315-322.

McClure, M.L. (1981). Promoting practice-based research: A critical need. Journal of Nursing Administration. 11:66-70.

Mercer, R.T. (1984). Nursing research: The bridge to excellence in practice. Image, 16(2):57-58.

Noble, M.A. (1980). Teaching clinical research: idealism versus realism. Journal of Nursing Education. 19:34-37.

Oberst, M.T. (1985). Integrating research and clinical practice roles. Topics in Clinical Nursing, 7:45-53.

O'Brien, D., Heyman, B. (1989). Changes in nurse education and the facilitation of nursing research; an exploratory study. Nurse Education Today, 9:392-396.

Poster, E.C., Betz, C.L., Randell, B. (1992). Psychiatric nurses' attitudes toward and involvement in nursing research. Journal of Psychosocial Nursing, 30(10):26-29.

Rettig, F.M. (1980). Nurses' attitudes toward research. Association of Operating Room Nurses Journal, 31(7):1251, 1254-1255.

Rizzuto, C., Bostrom, J., Suter, W.N., Chenitz, W.C. (1994). Predictors of nurses' involvement in research activities. Western Journal of Nursing Research, 16(2):193-204.

Robichaud-Ekstrand, S., Sherrard, H. (1994). Cardiac nurses' perceptions of nursing research. Progress in Cardiovascular Nursing, 9(3):7-15.

Schlotfeldt, R.M. (1992). Why promote clinical nursing scholarship? Clinical Nursing Research, 1(1):5-8.

Smeltzer, C.H., Hinshaw, A.S. (1988). Research: clinical integration for excellent patient care. Nursing Management, 19(1):38-41.

Statistique Canada (1993). Données sur les infimiers(ères) autorisé(e)s à l'intention de la direction. Division des statistiques sur la santé.

Thomas, E. (1985). Attitudes towards nursing research among trained nurses. Nurse Education Today, 4(4):18-21.

Vail, S., Hicks, S.E. (1996). Issues \& trends. The growing importance of health research. Canadian Nurse, 92(3):59-60.

Varcoe, C., Hilton, A. (1995). Factors affecting acute-care nurses' use of research findings. Canadian Journal of Nursing Research, 27(4):51-71.

Webb, C, MacKenzie, J. (1993). Where are we now? Research-mindedness in the 1990s. Journal of Clinical Nursing, 2:129-133.

Wong, C. (1996). Impact of restructuring and redesign on nursing research-based practice in service setting. Nursing Research Interest Group News, 10(2):1-2. 